\title{
Context Adaptation of Fuzzy Inference System-Based Construction Labor Productivity Models
}

\author{
Abraham Assefa Tsehayae ${ }^{1}$ and Aminah Robinson Fayek ${ }^{2}{ }^{2}$ \\ ${ }^{1}$ School of Civil and Environmental Engineering, Addis Ababa Institute of Technology, Addis Ababa University, Room 206, \\ AAiT Main Building, P.O. Box 385, Addis Ababa, Ethiopia \\ ${ }^{2}$ Department of Civil and Environmental Engineering, Hole School of Construction Engineering, University of Alberta, \\ 7-287 Donadeo Innovation Centre for Engineering, Edmonton, AB, Canada T6G 1 H9
}

Correspondence should be addressed to Aminah Robinson Fayek; aminah.robinson@ualberta.ca

Received 21 January 2018; Accepted 29 March 2018; Published 14 May 2018

Academic Editor: Stella Morris

Copyright (C) 2018 Abraham Assefa Tsehayae and Aminah Robinson Fayek. This is an open access article distributed under the Creative Commons Attribution License, which permits unrestricted use, distribution, and reproduction in any medium, provided the original work is properly cited.

Construction labor productivity (CLP) is one of the most studied areas in the construction research field, and several contextspecific predictive models have been developed. However, CLP model development remains a challenge, as the complex impact of multiple subjective and objective influencing variables have to be examined in various project contexts while dealing with limited data availability. On the other hand, lack of a framework for adapting existing or original models from one context to other contexts limits the possibility of reusing existing models. Such challenges are addressed in this paper through the development of a context adaptation framework. The framework is used to transfer the knowledge represented in fuzzy inference (FIS) based CLP models from one context to another, by using linear and nonlinear evolutionary based transformation of the membership functions combined with sensitivity analysis of fuzzy operators and defuzzification methods. Using four context-specific CLP models developed for concreting activity under industrial, warehouse, high-rise, and institutional building project contexts, the framework was implemented, and the prediction capability of the adapted models was evaluated based on their prediction similarity with the original models. The results showed that linearly adapted CLP models for industrial and institutional contexts and nonlinearly adapted CLP models for warehouse and high-rise contexts provide a similar prediction capability with the original models. The proposed context adaptation framework and findings from this paper address the limitations in past context adaptation research by examining a practical context-sensitive application problem and further examining the role of fuzzy operators and defuzzification methods. The findings assist researchers and industry practitioners to take full advantage of existing FIS-based models in the study of new contexts, for which data availability might be limited.

\section{Introduction and Background}

Construction labor productivity (CLP) has a direct and significant influence on success of construction projects; therefore, CLP has been well studied [1]. As a result, numerous predictive CLP models have been tested and developed, even though, collecting and modeling productivity data are known to require significant financial investment [2]. Existing CLP models have used various data analysis methods, including regression analysis, neural networks, expert systems, and fuzzy inference systems [1]. CLP modeling deals with a complex problem involving a large number of subjective and objective variables and is faced with limited data availability, thus, making CLP modeling an exceptional target for fuzzy inference systems. Fuzzy inference systems (FISs) have been proven to be effective tools for solving many engineering problems in biomedicine, robotics, pattern recognition, image processing, and control application areas [3]. More importantly, FISs are suitable for context adaption, whereby they can be adapted to suit other project contexts and enable users to take full advantage of existing FIS in the analysis of new contexts. Such a context adaptation process cannot 
be utilized with other CLP modeling approaches such as regression equations or neural networks, as the existing models have to be redeveloped or retrained before use in new contexts. Mao [4] used FIS to model labor productivity of concrete wall formwork activity. Pan [5] studied the effect of rainfall on productivity and duration of highway activities using a FIS. Fayek and Oduba [6] also used FISs to model the labor productivity of industrial pipe rigging and welding activities. Tsehayae and Fayek [7], using a system model approach, studied CLP of concreting activity under industrial, warehouse, high-rise, and institutional building project contexts; the authors used a fuzzy hybrid approach, which incorporated FISs, developed using a data-driven fuzzy clustering technique, combined with a genetic algorithm-based model tuning process. However, model input variables (i.e., CLP influencing variables) used in the past FIS-based CLP models and their associated impact on CLP varied from project to project, thus, making all of the developed CLP models context-specific.

Context plays an essential role in FIS-based CLP research, as it defines in which scenarios the findings of the FISbased CLP models are applicable, which implies that, under changed contexts, existing models cannot be used without some adaptation. Moreover, context adaptation of FISs has been recognized as an effective method for generating interpretable and accurate FIS models through tuning of the parameters contained in the data sets and based on the collected context-specific information [8]. However, in the CLP modeling field, an approach for adapting such models from one context to another context is missing. Model adaptation is of particular importance for Mamdani-type FIS-based models, as the fuzzy sets used in such models are highly context-specific. In FIS, the if-then rules are composed of fuzzy conditions (represented by the MFs of the input variables) and fuzzy conclusions (represented by the MFs of the output variables). Fuzzy sets are used to describe the respective linguistic variables, which characterize a CLP influencing variable like crew size using linguistic variables such as small crew size, medium crew size, and large crew size. An example of a CLP focused fuzzy rule is shown in the following, where the words in italics are the influencing variables or features and the linguistic variables are shown in bold. "If the crew size is small and the cooperation among craftsperson is very good and the level of interruption and disruption is low and the direct work proportion is high then construction labor productivity is high."

A fuzzy set $A$, representing a linguistic variable, is characterized using its membership function (MF), which represents numerically the degree to which an element $x$ belongs to the fuzzy set and fits the linguistic variable over a continuous range $A: \mathbf{X} \rightarrow \mu=[0,1]$. In the FISbased CLP models, the input (key factors, practices, and work sampling proportions) and output (CLP) variables are partitioned using linguistic variables over their respective universe of discourse. For example, the linguistic variables could be represented using Gaussian membership functions. The function for a Gaussian $\operatorname{MF} A(x, \sigma, \mu)$ is shown in (1), where $\sigma$ represents the standard deviation, denoting the spread of $A$, and $\mu$ represents the modal value, denoting the typical element of $A$ :

$$
A(x, \sigma, \mu)=e^{-(x-\mu)^{2} / 2 \sigma^{2}} .
$$

Membership functions (MFs) have many important descriptors. MFs employed in modeling endeavours are required to be normal; that is, at least one element of $\mathbf{X}$ attains full membership $(\mu=1)$ and represents a typical value of the fuzzy set. Support of fuzzy set $A$ represents all elements of $\mathbf{X}$ that exhibit some association with the fuzzy set by having nonzero membership degrees and core of fuzzy set $A$ represents all elements of the universe $\mathbf{X}$ that are typical to $A$. Fuzzy inference systems consist of two main parts: a rule-base (RB) composed of the linguistic ifthen rules and a database (DB) which associates membership functions to the linguistic variables used in the $R B$, for example, small for crew size or high for CLP. However, it is worth noting that, in the DB parts of FISs, the concept of "small crew size" cannot be uniquely and universally defined, while concepts like "crew size under 5 " can be. It is natural that construction professionals could define such fuzzy sets differently depending on the context of use, and the context itself must be properly defined using context attributes such as the contractor organization's experience, project location, and type of projects (e.g., commercial or industrial). Thus, the exact definition of a fuzzy set like small crew size depends on a context attribute like type of project [9]. For example, industrial projects tend to have larger crew sizes as compared to commercial ones; thus, a small crew size in an industrial project could be equivalent to a medium crew size in commercial projects. On the other hand, the rulebase is assumed to be context-free and is usually valid in any context, as the context of use affects only the meaning associated with each linguistic term used in the rules rather than the logic of the rules themselves $[8,10]$. Therefore, in the context adaptation of FISs, the focus is on the linguistic variables and their respective membership functions.

Accordingly, the main objectives of this research are to (1) develop a framework for context adaption of FIS-based CLP models, (2) test the framework using real-world problem, and (3) improve on limitations with existing context adaptation approaches which relied on normalized MFs. This objective is critical in CLP research domain as context adaptation will enable the reuse of existing FIS models in new contexts, thereby saving model developers the considerable effort required to collect data and to develop new CLP models; it will also improve the application of existing models by construction organizations as existing models can be adapted to suit the organizations' specific needs or contexts.

This paper begins with a literature review of the general context adaptation approaches. Then, a framework for adapting CLP models is formulated. Next, the paper illustrates the application of the framework by adapting a series of contextspecific CLP models to suit new contexts based on the field data collected for the same model features (i.e., model input and output variables) of the respective original CLP models. Then, a comparative assessment in terms of prediction accuracy and similarity between the adapted and original 
context-specific models is carried out, and the effectiveness of the context adaptation framework is discussed. Finally, conclusions and areas for future research are presented.

\section{Literature Review of Context Adaptation Approaches}

Context plays an active part in construction research analysis as it is invariably dynamic and imperative for the development of meaningful findings [11]. Thus, context adaptation has an important application in deriving new models from existing ones. In the general computing field, most of the context adaptation research has been carried out on Mamdani-type FISs, which have been used in a wide range of areas due to their ability to handle linguistic concepts and perform accurate modeling of input-output relations [3]. In the context adaptation of FISs, the focus has been mainly on the linguistic variables and their respective membership functions as the rule-base is considered to be a context-free model $[12,13]$. According to Botta et al. [8], the following principles have been followed in adapting FISs: (1) context adaptation will not modify the rule-base as the rule-base is considered to be a context-free and universal knowledge; (2) context adaptation will not change the number of linguistic variables and, consequently, the number of corresponding fuzzy sets defined in the rule-base; and (3) context adaptation will not affect the sematic ordering of linguistic variables.

Context adaptation of FISs has been carried using either transformation functions or adaptive operators. Most context adaptation studies focused on the use of transformation functions [12-15]. According to Botta [3], a transformation function serves to adapt a database (DB) or fuzzy partition, which is made up of a group of MFs, each representing a linguistic term. The transformation function maps the universe of discourse of the base or original context MF to the universe of discourse of the adapted context, thereby modifying the distribution (i.e., support and core) and the shape of the MFs. Transformation functions have been commonly applied to adapt the base partition defined over a normalized universe of discourse $[0,1]$ as shown in Figure 1. However, the use of a normalized universe of discourse for the base or original MFs makes the database more general or context independent as the MFs in the DB are defined over a normalized $[0,1]$ range $[12,13]$. In such a case, the universe of discourse is partitioned using common MFs like triangular, trapezoidal, or Gaussian [15] and is then adapted to another context partition defined over a universe of discourse of $[a, b]$ as shown in Figure 1. Commonly, linear and nonlinear transformation functions are used. Linear transformation functions are applied on the overall partition of the fuzzy sets and will either linearly expand or contract all the fuzzy sets (see Figure 1). However, nonlinear transformation functions are applied either to the overall partition or selected points of the fuzzy sets (refer to Figure 1), thus, changing all fuzzy sets or the breakpoints (i.e., points of intersection) of selected fuzzy sets [8].

The parameters of nonlinear transformation functions are derived using genetic algorithm or neural network based optimization approach over data collected for the new or target context using either field experiments or experts $[12,13]$. Several nonlinear transformation functions have been applied. Magdalena [14] proposed a sign function for nonlinear transformation of fuzzy sets and provided an application example using cart-pole balancing system control problem. Gudwin et al. [12] used a linear combination of sigmoidal functions and demonstrated the development of the functions using assumed data. Pedrycz and Gomide [16] suggested the use of piecewise linear function. According to Gudwin et al. [12], transformation functions should meet certain requirements so as to preserve ordering and normality of the base linguistic variables. In particular, they are required to fulfil continuity, nondecreasing monotonicity, and boundary conditions.

Adaptive operators are specifically designed operators that adjust the universe of discourse of the fuzzy sets and modify the core, support, and shape of fuzzy sets. According to Botta [3], the context adaptation process using adaptive operators is based on a flexible nonlinear transformation function and four orthogonal fuzzy modifiers: core-position modifier, core-width modifier, support-width modifier, and membership function shape modifier. The fuzzy modifiers are formulated using genetic algorithm based optimization process. Botta [3] tested the approach using four datasets, including a context-aware benchmarking dataset that arbitrarily assigns MFs to a universe of discourse, structure of wage dataset which includes years of experience and wage, a synthetic dataset generated using a parametric function, and fuel consumption dataset. However, adaptive operators modified the fuzzy partition in way that the order of the fuzzy sets was affected, resulting in reduced interpretability of the adapted fuzzy partition [8].

The interpretability of the adapted fuzzy partitions can be verified using coverage, normality, and distinguishability properties [8]. The adapted fuzzy partition has to cover the new or adapted context's universe of discourse $U=[a, b]$. The adapted MFs of the fuzzy sets are also required to be normal so that at least one element of the universe of discourse will have full membership. The overlap of the fuzzy sets in the adapted fuzzy partitions has to be kept to a level that each couple of fuzzy sets are distinguishable enough [3]. The overlap between fuzzy sets $\left(A_{1}, A_{2}\right)$ can be measured using a possibility measure $\Pi$ (see (2)). In order to ensure interpretability of the adapted fuzzy partition, the overlap $(\Pi)$ among the fuzzy sets should not exceed 0.8 [17].

$$
\Pi\left(A_{1}, A_{2}\right)=\sup _{x \in U} \min \left\{\mu_{A_{1}}(x), \mu_{A_{2}}(x)\right\} .
$$

In the general computing field, several FIS context adaptation studies have been carried out $[8,12,14,18]$; however, the following limitations are observed. First, transformation function based studies were applied on normalized base or original MFs defined over $[0,1]$ range, resulting in the adaptation of theoretical or context independent MFs to contexts-specific MFs. Second, most studies lacked practical application and rather focused on demonstrating the context adaptation method using benchmark datasets. Third, in FISs, not only the MFs but also the fuzzy operators and defuzzification methods are context-dependent [19]. However, past 


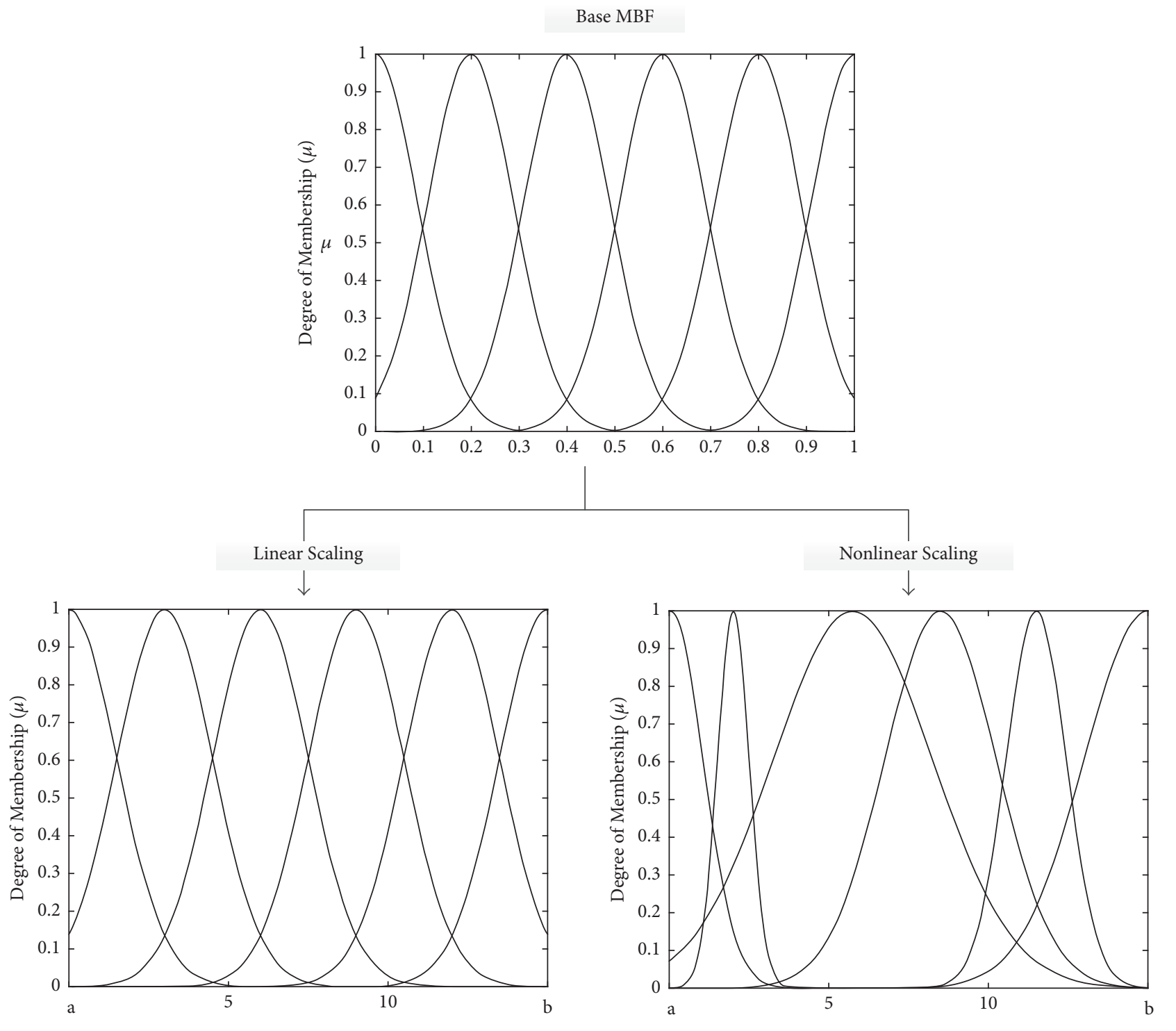

FIGURE 1: Fuzzy inference system context adaptation using transformation functions.

studies mainly used minimum operator for input aggregation and implication and centroid for defuzzification [3] and failed to evaluate the sensitivity of adapted models for fuzzy operators and defuzzification methods.

Similarly, in the construction research field, context adaptation is also scantly explored. Past studies have not studied context adaptation of FIS models as an approach for reusing existing models in new contexts but rather focused on the fine tuning of the FIS model parameters with the sole aim of improving the model's accuracy. According to Awad and Fayek [20], the fine tuning of a FIS model improved the accuracy of predicting contractor's default by tuning the MFs and weight of rules of the FIS using neural network and genetic algorithm techniques. Fayek and Oduba [6] improved the accuracy of FIS-based CLP models for industrial pipe rigging and welding activities, by shifting the right, left, and both legs of the triangular and trapezoidal output MFs. Idrus et al. [21] also followed the FIS tuning approach proposed by Fayek and Oduba [6] and showed an improvement in accuracy of predicting construction project cost contingency.

\section{Context Adaptation of CLP Models Using Transformation Functions}

This paper provides a framework that addresses CLP modeling challenges through the use of a context adaptation framework. The context adaptation framework is based on linear and nonlinear transformation functions rather than adaptive operators, as transformation functions provide a transparent context adaptation framework for the MFs of FIS-based CLP models while adaptive operators will modify the fuzzy partition, resulting in reduced interpretability of the adapted fuzzy partition. The framework, unlike past studies which focused on context independent base MFs, will focus 

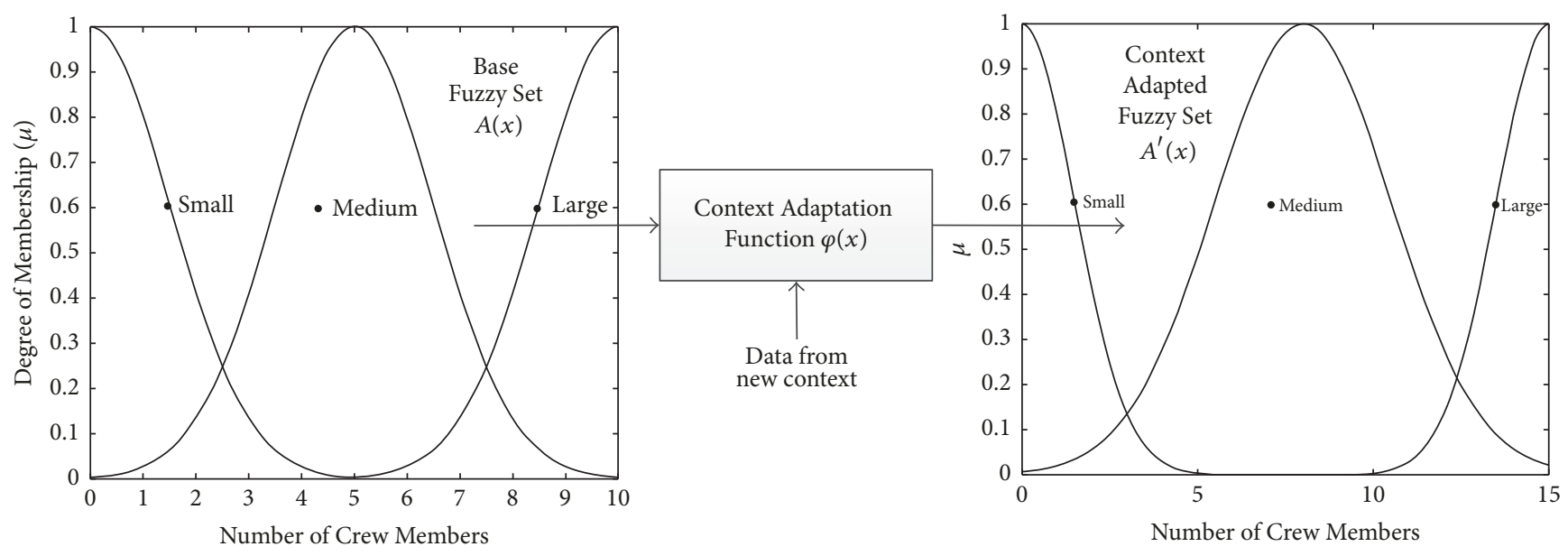

FIGURE 2: Context adaptation procedure.

on adapting context-specific MFs that are defined over universe of discourse $B=[l, u]$ to another context defined over a universe of discourse $U=[a, b]$. In addition to adapting the MFs, the framework also tests the effect of fuzzy operators and defuzzification methods on the adapted models. The following procedure is developed for context adaptation of FIS-based CLP models. The underlying process in the context adaptation procedure involves the determination of a context adaptation or transformation function, using data collected from the new context, for adapting the base fuzzy set $A$, represented by the base or base $\mathrm{MF}$, to an adapted fuzzy set $A^{\prime}$, represented by the adapted MF as shown in Figure 2.

The procedure for context adaptation is summarized in the following steps:

(1) Identify the base fuzzy sets for a given context and for each model feature (model input and output variables) of the base context-specific CLP model. The universe of discourse of each feature $B=[l, u]$ and the parameters of the MFs such as the standard deviation and modal values of Gaussian MF's of the base fuzzy set $A(x, \sigma, \mu)$ are documented.

(2) Collect data from the new context for each feature of the base model, using field data collection or experts. The documented data set $\left(d_{1}, d_{2}, \ldots, d_{N}\right)$, where $N$ represents the total number of data instances of a given feature, will then be used to determine the upper and lower limits of the adapted fuzzy set $A^{\prime}(x)$, the preliminary MFs of the adapted fuzzy set $A_{0}^{\prime}(x)$, and then the appropriate context adaptation function $\varphi_{h}(x)$.

(3) Determine the boundary or upper and lower limits of the adapted fuzzy set $U=[a, b]$. For example, using the absolute limit context determination approach [12], the lower bound $a$ is taken as the minimum data value; thus $a=\min \left(d_{1}, d_{2}, \ldots, d_{N}\right)$ and the upper bound $b$ is taken as the maximum data value; thus $b=\max \left(d_{1}, d_{2}, \ldots, d_{N}\right)$.

(4) Determine the preliminary MFs of the adapted fuzzy set $A_{0}^{\prime}(x)$ using the collect data from the new context using either expert- or data-driven MF development approaches. Expert-driven approaches such as direct (horizontal or vertical), exemplification, and pairwise comparison or data-driven approaches such as inductive reasoning and fuzzy clustering can be used. The preliminary MFs will be used to determine the nonlinear transformation function.

(5) Determine the parameters of the context adaptation functions $\varphi_{1}(x)$ for linear and $\varphi_{2}(x)$ for nonlinear transformation of MFs.

(6) Develop the MF of the adapted fuzzy set $A^{\prime}(x)$ using either the linear or nonlinear adaptation functions as expressed in

$$
\begin{aligned}
& A_{i}^{\prime}(x)=A\left(\varphi_{h}(x)\right), \quad x \in[a, b], \\
& \varphi_{h}(x) \in[l, u], \quad h \in\{1,2\} .
\end{aligned}
$$

(7) Evaluate the overlap among the adapted MFs using possibility measure (see (2)) in order to ensure the distinguishability of adapted fuzzy sets.

(8) Adapt the base CLP model by replacing the base MFs $A(x)$ with the adapted MFs $A^{\prime}(x)$ for each model feature (input and output variables) and evaluate the prediction ability of the adapted CLP model. FIS model accuracies can be determined using the root mean square error (RMSE) [22]. The RMSE of the adapted CLP model is calculated using (4), where $t_{i}$ is the new context's target CLP value for the $i$ th data instance, $z_{i}$ is the corresponding predicted CLP value, and $N$ is the total number of data instances.

$$
\operatorname{RMSE}_{i}=\frac{1}{N} \sum_{i=1}^{N} \sqrt{\left(t_{i}-z_{i}\right)^{2}} .
$$

(9) Evaluate the sensitivity of the adapted CLP model for fuzzy operators and defuzzification methods and summarize the improvement in prediction ability. 
(10) Determine the similarity between the adapted and base model of the new context using model agreement measures. The use of the modified Willmott agreement index is recommended to determine the similarity between models. The index is dimensionless, bounded, less sensitive to extreme values and outliers, and suitable for cross-comparison between models [23]. The Willmott agreement index $\mathrm{WI}_{i}$ is shown in (5), where $\mathrm{PA}_{i}$ represents the predicted values obtained from the adapted model, $\mathrm{PB}_{i}$ is the predicted values obtained from the base or original model of the new context, $\overline{\mathrm{PB}}$ is the mean value of the predicted values from the base model of the new context, and $N$ is the total number of data instances. The value $\mathrm{WI}_{i}$ varies from 0 to 1 , and a value of 1 indicates a perfect agreement between the adapted and base models.

$$
\mathrm{WI}_{i}=1-\frac{\sum_{i=1}^{N}\left|\mathrm{PA}_{i}-\mathrm{PB}_{i}\right|}{\sum_{i=1}^{N}\left(\left|\mathrm{PA}_{i}-\overline{\mathrm{PB}}\right|+\left|\mathrm{PB}_{i}-\mathrm{PB}\right|\right)} .
$$

(11) Compare and contrast the agreement indices and prediction ability of the resulting adapted models for both linear and nonlinear transformation of MFs and combinations of fuzzy operators and defuzzification methods and identify the most appropriate context adaptation approach.

\section{Context Adaptation of Context-Specific Concreting Activity CLP Models}

The preceding context adaptation procedure was tested using the field data collected for concrete pouring activity. The data were gathered from six building projects in the greater Edmonton area of Alberta, Canada, from June 2012 to October 2014. The projects included (1) a mixed-use office and staff facility building, (2) an industrial warehouse building, (3) a commercial warehouse building, (4) a mixed residential and community center building, (5) a mixed commercialresidential building, and (6) an institutional building. Concrete pouring activity was studied in three data collection cycles, where each cycle extended over a month-long period and encompassed different weather seasons. For each data collection case, WS observations were made for the crew under study, and influencing variables (factors and practices), total man-hours, and installed quantities were documented. CLP was defined as the ratio of units of output-in terms of installed quantity $\left(\mathrm{m}^{3}\right)$ - to units of input-in terms of total labor work hours $(\mathrm{MH})$ - where higher CLP values are desirable.

First, an operational definition of context for CLP modeling was developed, which was based on the context attributes that constrain the four elements of a CLP model (user, model developer, model, and prevailing environment of the model) without intervening in the model development process explicitly. Accordingly, the context attributes for each of the studied six projects were generated using the $5 \mathrm{~W} 1 \mathrm{H}$ questions approach: Who, What, Where, When, Why, and
How. Among the generated context attributes, the following context attributes distinguished the six projects that were studied and, thus, were used as the key context attributes in comparing and identifying the similarity of the projects: "Who" attributes, related to the project owner's primary driver (schedule, cost, quality, or safety), contractor team's experience, and contractor organization's experience; "What" attributes related to project (i.e., building) type, site layout, project safety practice, and project productivity measurement and tracking practice; and "Where" attributes related to project location.

Accordingly, projects having identical values to key context attributes were grouped together, and the project nature (building type) context variable was used to name the four unique contexts. Context 1 , representing concreting in industrial buildings, includes the datasets of the first two projects (the mixed-use office and staff facility building and the industrial warehouse building). Context 2, representing concreting in Warehouse buildings, includes the dataset of the third project (a commercial warehouse building). Context 3 , representing concreting in high-rise buildings, includes the datasets of the fourth and fifth projects (the mixed residential and community center building and the mixed commercial-residential building). Context 4, representing concreting in institutional buildings, includes the data set of the sixth project (the institutional building). A more detailed discussion of the context attributes can be found in the study presented in the thesis work of the first author [24].

Then, four context-specific CLP models, summarized in Table 1, were developed and optimized for predicting labor productivity of concreting (concrete pouring) activity using Mamdani-type FIS models, which were used as base or original CLP models. The models were developed using Fuzzy C-Means clustering and Gaussian membership functions (MFs) were used. The following FIS model parameters were optimized: (1) the fuzzification coefficient $m$ in FCM clustering, (2) membership function parameters, (3) number of rules, and (4) fuzzy operators and defuzzification methods, resulting in improved accuracy and interpretability of the four context-specific CLP models. A more detailed discussion of the context-specific model development process can be found in the study presented by Tsehayae and Fayek [7].

The properties of the optimized FIS-based CLP models, shown in Table 1, indicate the context-specific nature of models as they had distinctly different key influencing input variables (made up of factors, practices, and work sampling proportions), number of membership functions or number of rules, fuzzification coefficients, fuzzy operators, and defuzzification methods. The context adaptation framework presented in this paper was tested by adapting the four context-specific CLP base models from one context to another, as shown in Figure 3. Accordingly, the industrial context CLP model is adapted to suit the warehouse, highrise, and institutional contexts using linear and nonlinear transformation functions, and a similar process is repeated for the other three contexts. The adaptation process, for each context, resulted in six adapted models: three linearly adapted models and three nonlinearly adapted models (see Figure 3). The adapted models are compared with the base 


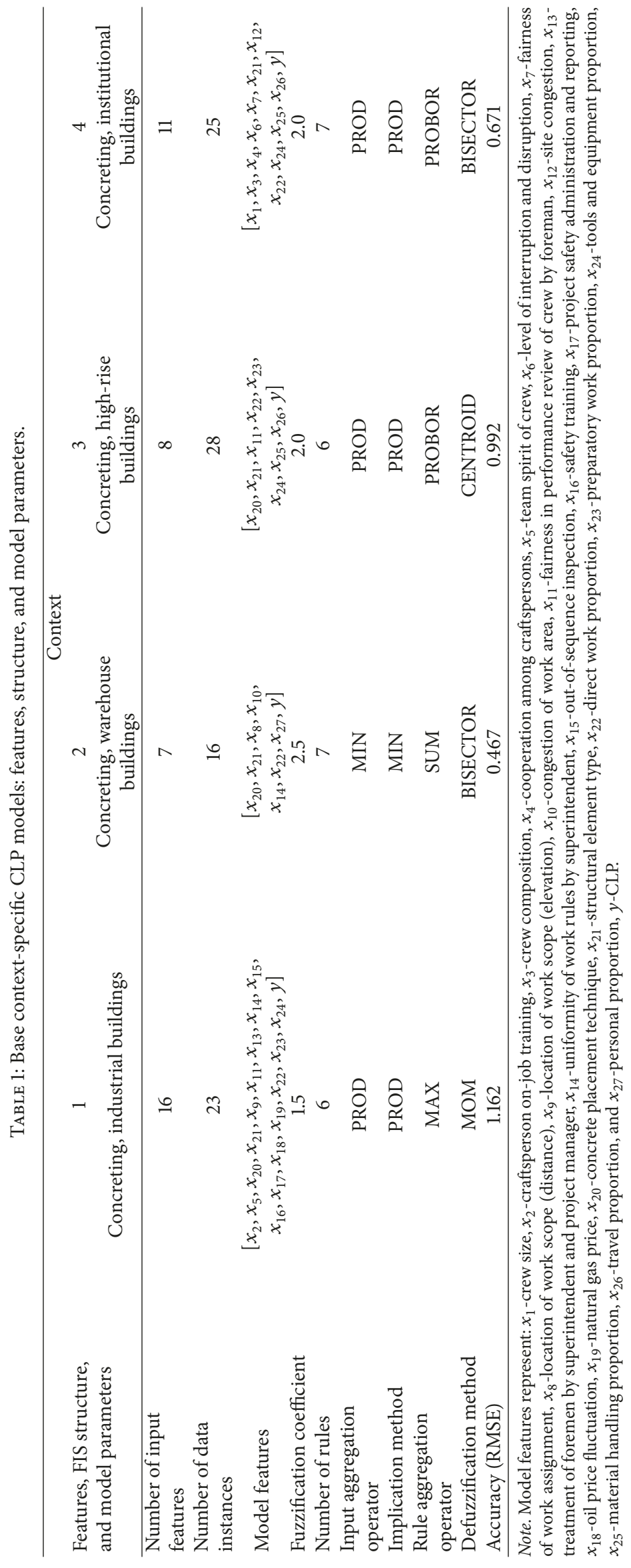




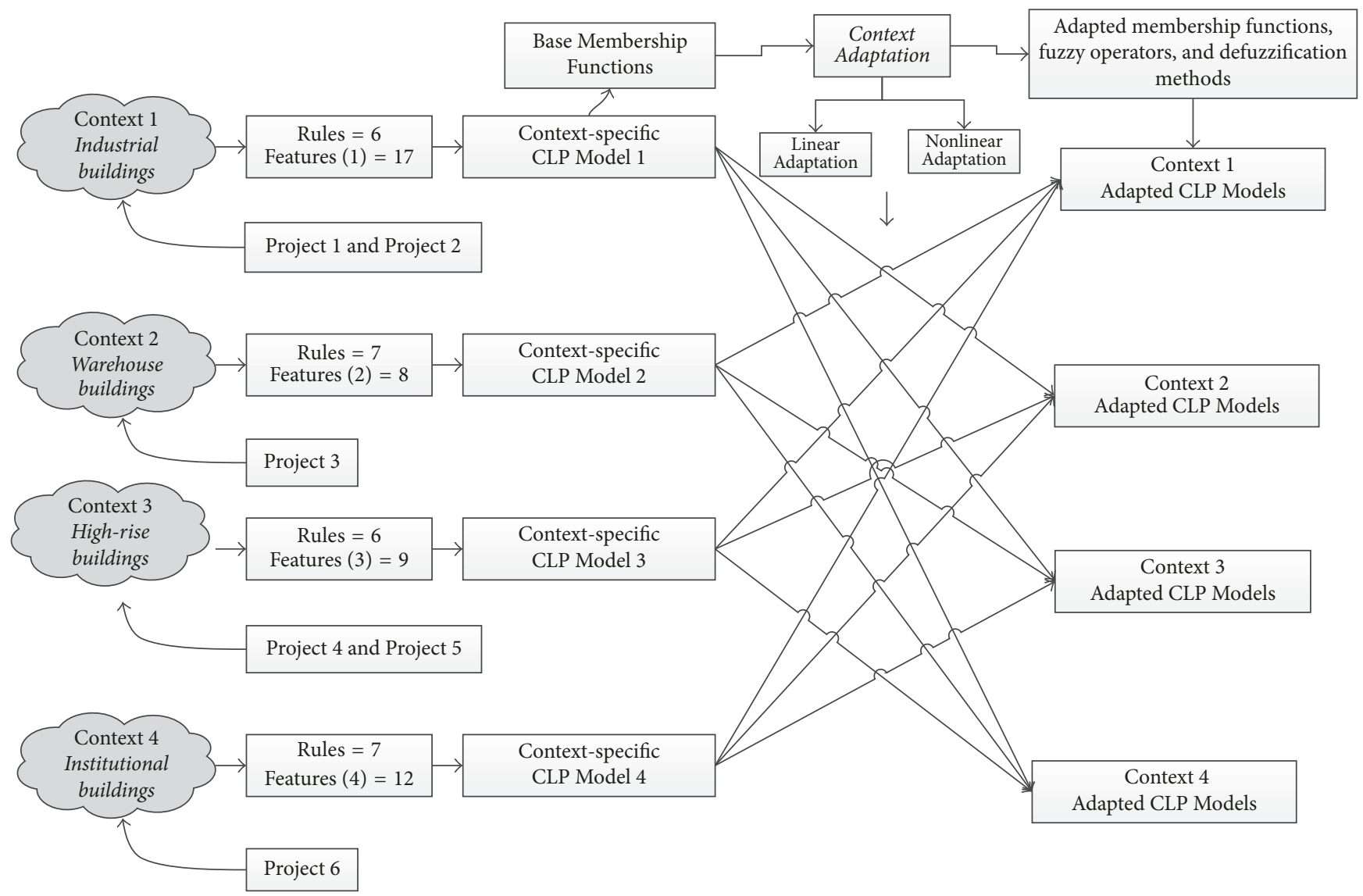

FIgURE 3: Context-specific CLP models adaptation.

model developed for the given context (refer to Table 1) using model accuracy in terms of RMSE and modified Willmott agreement indices.

In the following sections, a numerical illustration of the context adaptation framework is discussed based on the adaptation of the model output (CLP) feature from institutional to industrial context. The institutional (context 4) CLP model has 12 model features (11 input and one model, i.e., CLP variable) and seven rules, implying an equal number MFs representing the fuzzy sets $A_{j}=\left\{A_{1}, A_{2}, \ldots, A_{7}\right\}$. The base MFs for model output (CLP) had a universe of discourse $B=[1.80,11.25]$ and the mean CLP was $4.25 \mathrm{~m}^{3} / \mathrm{MH}$, with standard deviation of 2.21 .

For given context-specific CLP model, all model features' MFs are adapted using linear and nonlinear transformation functions to three other contexts, and initial model accuracies, $\mathrm{RMSE}_{\mathrm{CA}-\mathrm{L}}$ (where CA-L represents the context adapted CLP model using linear adaptation) and $\mathrm{RMSE}_{\mathrm{CA}-\mathrm{NL}}$ (where CA-NL represents the context adapted CLP model using nonlinear adaptation), respectively, are established. Then, the sensitivity of the adapted models for fuzzy operators and defuzzification methods is investigated for both linear and nonlinear adapted models and final model accuracies, RMSE $_{\text {CA-LS }}$ (where CA-L represents the context adapted CLP model using linear adaptation after sensitivity analysis) and $\mathrm{RMSE}_{\mathrm{CA}-\mathrm{NLS}}$ (where CA-L represents the context adapted
CLP model using nonlinear adaptation after sensitivity analysis), respectively, are established. Next, the agreement indices $\left(\mathrm{WI}_{i}\right)$ between adapted and base models were computed for identifying the appropriate linear or nonlinear adaptation approach. Finally, the model accuracies of the adapted models are compared and the best performing context adaptation approach is identified. For the adapted MFs the following linguistic labels are used: $A_{1}$ (very low), $A_{2}$ (low), $A_{3}$ (medium low), $A_{4}$ (medium), $A_{5}$ (medium high), $A_{6}$ (high), and $A_{7}$ (very high). The parameters of the seven Gaussian MFs $A(x, \sigma, \mu)$ of the base or base model output variable are as follows: $A_{1}(x)=G(x, 0.973,1.875), A_{2}(x)=G(x$, $0.573,3.514), A_{3}(x)=G(x, 0.786,4.569), A_{4}(x)=G(x$, $0.864,5.990), A_{5}(x)=G(x, 0.877,7.673), A_{6}(x)=G(x$, $0.707,9.101)$, and $A_{7}(x)=G(x, 1.101,11.105)$.

The CLP data from industrial context (context 3) was then retrieved from collected data and had values of universe of discourse $U=[0.03,8.66]$ and the mean CLP was $2.69 \mathrm{~m}^{3} / \mathrm{MH}$, with standard deviation of 1.83 . Next, the parameters of the context adaptation functions $\varphi_{1}(x)$ for linear adaptation and $\varphi_{2}(x)$ for nonlinear adaptation are developed, as discussed in the following subsections.

4.1. Linear Adaptation. In linear adaptation, the base MFs defined over a universe of discourse of $B=[l, u]$ are adapted to the context-adapted universe of discourse by means of a 

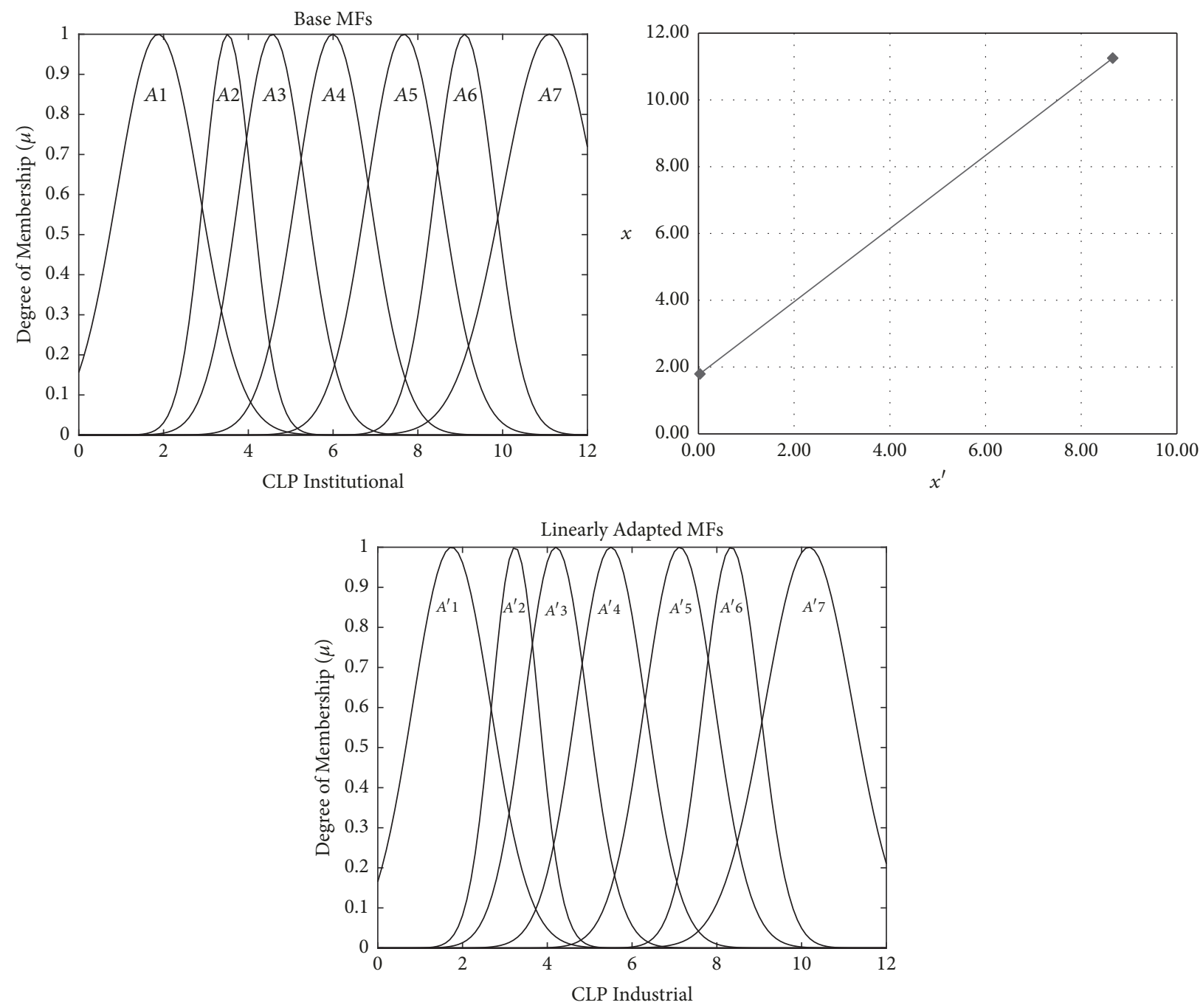

FIGURE 4: Linear context adaptation of CLP feature from Institutional to industrial context.

linear transformation function shown in (6), where $U=[a, b]$ is used to represent the bounds of the adapted MF:

$$
\varphi_{1}(x, a, b)=\frac{(b-a)}{(u-l)} x+a .
$$

Accordingly, the respective institutional CLP context linguistic variables $A(x)$ are adapted to industrial CLP context linguistics variables $A^{\prime}(x)$ using the linear transformation function shown in the following:

$$
\varphi_{1}(x)=x^{\prime}=\frac{(8.66-0.03)}{(11.25-1.80)} x+0.03=0.913 x+0.03 .
$$

Thus, the seven MFs $A^{\prime}(x)$ for CLP feature in the adapted context (industrial context) are determined by replacing the parameters of the membership function $[\sigma, \mu]$ of the base fuzzy sets $A_{j}=\left\{A_{1}, A_{2}, \ldots, A_{c}\right\}$ with adapted values based on $\varphi_{1}(x)$. The parameters of the seven adapted Gaussian MFs $A^{\prime}\left(x, \sigma^{\prime}, \mu^{\prime}\right)$ of the CLP variable are $A^{\prime}{ }_{11}(x)=G(x, 0.918$, 1.742), $A^{\prime}{ }_{12}(x)=G(x, 0.553,3.239), A_{13}^{\prime}(x)=G(x, 0.748$, 4.203), $A_{14}^{\prime}(x)=G(x, 0.819,5.500), A_{15}^{\prime}(x)=G(x, 0.831$,
7.123), $A^{\prime}{ }_{16}(x)=G(x, 0.676,8.341)$, and $A^{\prime}{ }_{17}(x)=G(x$, $1.035,10.171)$.

The results of the linear adaptation of the MFs are shown in Figure 4. The degree of overlap among the adapted MFs is not exceeding the limiting value of 0.8 [17]. A similar linear adaptation procedure was applied to the other 11 input variables of the Institutional CLP model.

Then, the MFs of the Institutional context model were replaced with the adapted ones, resulting in the linearly adapted Institutional context model for use in industrial context. The linearly adapted CLP model was used to predict CLP values of the industrial context, which had 23 data instances (refer to Table 1). The adapted model had an initial RMSE $_{\text {CA-L }}$ value of 1.832 .

4.2. Nonlinear Adaptation. Nonlinear adaption involves the use of a nonlinear transformation function that changes the universe of discourse of the base or base MFs and also modifies the shape and distribution of the MFs in the space of the adapted universe of discourse [3]. Accordingly, the base or base MFs defined over a universe of discourse of $B=[l, u]$ 


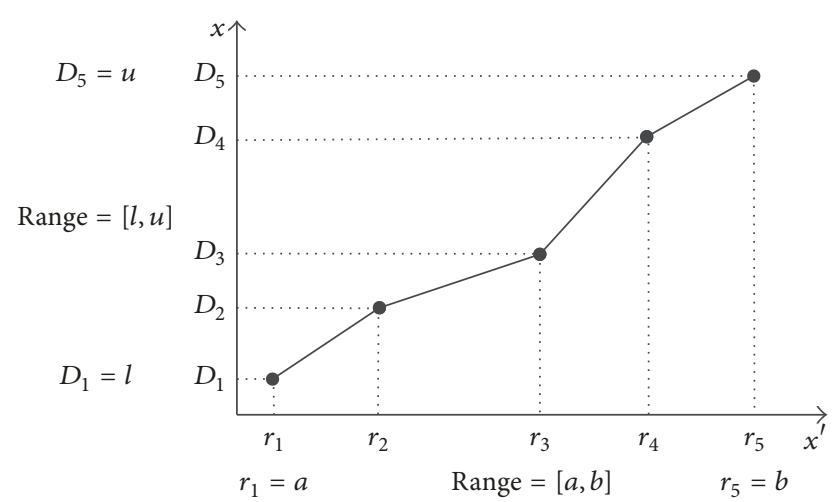

FIGURE 5: Nonlinear context adaptation function: $\varphi_{2}$.

are adapted in the context-adapted universe of discourse by means of a nonlinear transformation function $\varphi_{2}$, where $U=$ $[a, b]$ represents the identified bounds of the adapted MFs (refer to Figure 5). The determination of the parameters of the nonlinear transformation function requires an optimization process [12].

In this research, a piecewise linear transformation function is used in order to develop an interpretable, logical, and fully invertible, where the inverse is also a function, nonlinear context adaptation process [16]. For piecewise linear transformation function $\varphi_{2}$, shown in Figure 5, the set of the adjustable parameters $\mathbf{p}$ is made up of a collection of the split points $r_{1}, r_{2}, \ldots, r_{5}$ and associated differences $D_{1}, D_{2}, \ldots, D_{5}$, represented as $\mathbf{p}=\left[r_{1}, r_{2}, \ldots, r_{5}, D_{1}, D_{2}, \ldots, D_{5}\right]$. The piecewise functions, which will be determined using an optimization process discussed below, will result in nonlinear mapping as some regions of $x$ will be contracted and some of them will be expanded, resulting in modification of the shape and distribution of the MFs in the space of the adapted universe of discourse [16].

In order to improve the effectiveness of the optimization process required for determining the parameters of the piecewise linear transformation function with limited data from new contexts, the number of split points is kept to five points, as setting a higher number will have required increased amount of data and on the other hand setting a lower number will reduce the nonlinearity of the function. According to Gudwin et al. [12], context transformation functions are expected to fulfil the following requirements: continuity, monotonicity, and boundary conditions. The use of specifically nondecreasing monotonic piecewise functions ensures that the meaning and order of the linguistic terms is not changed [10]. Additionally, setting the boundary conditions $\varphi_{2}(l)=a$ and $\varphi_{2}(u)=b$ allows for the coverage of the new context data.

4.2.1. Computation of Nonlinear Transformation Function. Once the format or type of the nonlinear transfer function $\varphi_{2}$ is selected, the determination of the parameters of $\varphi_{2}$ in terms of $\mathbf{p}=\left[r_{1}, r_{2}, \ldots, r_{5}, D_{1}, D_{2}, \ldots, D_{5}\right]$ was carried out via optimization computations. The optimization process begins with the collection of MFs (linguistic terms)
$A_{j}=\left\{A_{1}, A_{2}, \ldots, A_{c}\right\}$ as the base MFs of a given model feature (model input or output variable) and the dataset $\left(d_{1}, d_{2}, \ldots, d_{N}\right)$ collected for the same feature, but from the new context. Then, using Fuzzy C-Means (FCM) clustering, the preliminary MFs representing the adapted fuzzy sets $A_{0}^{\prime}=$ $\left\{A^{\prime}{ }_{10}, A^{\prime}{ }_{20}, \ldots, A_{c 0}^{\prime}\right\}$ were developed using the collected dataset $\left(d_{1}, d_{2}, \ldots, d_{N}\right)$. The numbers of prototypes or cluster centres for FCM clustering are set equal to the number of base or base MFs, and the commonly used fuzzification coefficient of 2.0 is used [16]. The resulting degrees of memberships of each data instance in the preliminary adapted fuzzy sets are arranged in the form of $N(c+1)$ - tuples, as shown below, where the $k$ th tuple consists of $d_{k}$ that denotes a point, under consideration, in adapted universe of discourse $x^{\prime}$ where $\mu_{k 1}, \mu_{k 2}, \ldots, \mu_{k c}$ are the numeric values of the corresponding membership degrees of $d_{k}$ in preliminary adapted fuzzy sets $A_{10}^{\prime}, A^{\prime}{ }_{20}, \ldots, A_{c 0}^{\prime}$, respectively:

$$
\begin{gathered}
\left(d_{1},\left(\mu_{11}, \mu_{12}, \ldots, \mu_{1 c}\right)\right) \\
\left(d_{2},\left(\mu_{21}, \mu_{22}, \ldots, \mu_{2 c}\right)\right) \\
\vdots \\
\left(d_{N},\left(\mu_{N 1}, \mu_{N 2}, \ldots, \mu_{N c}\right)\right) .
\end{gathered}
$$

Then, the difference between the degree of membership of $d_{k}$ from the preliminary adapted MF and the degree of membership of $d_{k}$ computed using the transformation process was computed for each MF and for the respective data instance. This difference between the preliminary adapted fuzzy sets and the adapted MFs developed using the nonlinear transformation functions formed the objective function of the optimization process. The objective function $Q$ was calculated using sum of squared differences as shown in the following:

$$
\begin{aligned}
Q(p)= & \sum_{i=1}^{c}\left(A_{i}\left(\varphi\left(d_{1}, \mathbf{p}\right)-\mu_{1 i}\right)\right)^{2} \\
& +\sum_{i=1}^{c}\left(A_{i}\left(\varphi\left(d_{2}, \mathbf{p}\right)-\mu_{2 i}\right)\right)^{2}+\ldots \\
& +\sum_{i=1}^{c}\left(A_{i}\left(\varphi\left(d_{N}, \mathbf{p}\right)-\mu_{N i}\right)\right)^{2}
\end{aligned}
$$

Thus, the determination of the nonlinear transformation function involved the minimization of the objective function with respect to parameters of $\mathbf{p}$. The solution of this constrained nonlinear minimization optimization problem can be effectively developed using genetic algorithm, as the objective function is nonlinear and nonconvex (has multiple feasible regions), as the use of the traditional gradient based optimization techniques, such as generalized reduced gradient approach, will only lead to local optimum solutions [12]. Accordingly, an optimization process using genetic algorithm was used, and the parameters of the nonlinear transformation function $\varphi_{2}=\mathbf{p}=\left[r_{1}, r_{2}, \ldots, r_{5}, D_{1}, D_{2}, \ldots, D_{5}\right]$ were used for real coding of the chromosome in the genetic 
optimization process. The objective of the genetic search was to minimize the objective function $Q$, and the fitness value of each solution was determined by calculating $Q$. Then, the genetic operations of reproduction, crossover, and mutation were performed. Each operation generated new sets of chromosomes, representing a new solution that meets the optimization constraints. The solution chromosomes are checked according to the following nonlinear context adaptation constraints. (1) The parameters of the nonlinear transformation function $\mathbf{p}$ must be greater than zero. (2) Boundary conditions for coverage of the new context data are defined over $U=[a, b]$ and using the base or base MF range of $B=[l, u]: r_{1}=l, r_{5}=u, D_{1}=a$, and $D_{5}=b$. (3) The $j$ th split value $r_{j}$ must not be greater than that of the $j+1$ th split value $r_{j+1}$. (4) The $j$ th difference value $D_{j}$ must not be greater than that of the $j+1$ th difference value $D_{j+1}$. In the genetic optimization process, initial population of 100 solutions was randomly generated, and mutation rate of 0.075 and stopping criteria based on convergence value of 0.0001 was used.

4.2.2. Computational Results for Nonlinear Adaptation of CLP Models. The numerical illustration of the nonlinear context adaptation of the output feature (CLP) from institutional to industrial context is discussed here. As discussed above, the seven base fuzzy sets $A_{j}=\left\{A_{1}, A_{2}, \ldots, A_{7}\right\}$ for CLP variable and associated MFs from institutional context were first documented. Next the CLP dataset $\left(d_{1}, d_{2}, \ldots, d_{N}\right)$ collected from the industrial context is retrieved and had values of universe of discourse of $U=[0.03,8.66]$. Using Fuzzy $C$ Means (FCM) clustering, the preliminary MFs of the adapted fuzzy sets $A^{\prime}{ }_{0}=\left\{A^{\prime}{ }_{01}, A^{\prime}{ }_{02}, \ldots, A^{\prime}{ }_{07}\right\}$ were developed, where the number of prototypes for FCM clustering was set at seven, equal to the original number of MFs. Then, using genetic algorithm based optimization, the parameters of the nonlinear piecewise transformation function $\mathbf{p}$ were developed, and the resulting parameters were $\varphi_{2}=\left[r_{1}=\right.$ $0.03, r_{2}=2.05, r_{3}=5.91, r_{4}=6.36, r_{5}=8.66, D_{1}=$ $\left.1.80, D_{2}=5.52, D_{3}=7.76, D_{4}=9.63, D_{5}=11.25\right]$.

Thus, the seven MFs for CLP variable in the adapted context (industrial context) $A^{\prime}{ }_{2 j}(x)=\left\{A^{\prime}{ }_{21}, A^{\prime}{ }_{22}, \ldots, A^{\prime}{ }_{2 c}\right\}$ were determined by replacing the parameters of the membership function $[\sigma, \mu]$ of the base fuzzy sets $A_{j}=\left\{A_{1}, A_{2}, \ldots, A_{c}\right\}$ with adapted values based on $\varphi_{2}(x)$. The results of the nonlinear adaptation of the MFs are shown in Figure 6.

The parameters of the seven nonlinearly adapted Gaussian MFs $A^{\prime}{ }_{2 j}\left(x, \sigma^{\prime}, \mu^{\prime}\right)$ of the CLP variable were $A^{\prime}{ }_{21}(x)=G\left(x^{\prime}, 0.528,1.018\right), A^{\prime}{ }_{22}(x)=G(x, 0.311,1.908)$, $A^{\prime 2}{ }_{23}(x)=G(x, 0.427,2.481), A_{24}^{\prime}(x)=G(x, 0.469,2.860)$, $A^{\prime}{ }_{25}(x)=G(x, 0.476,5.760), A_{26}^{\prime}(x)=G(x, 0.384,6.233)$, and $A^{\prime}{ }_{27}(x)=G(x, 0.598,8.454)$. The results shown in Figure 6 indicate that the shape of some of the MFs has contracted, the distribution of the MFs over the universe of discourse have been modified, and in some cases the degree of overlap among the adapted sets was higher than the recommended maximum value of 0.8 [17]. Such changes of MFs will naturally reduce the interpretability of the adapted models; however, this is a common problem witnessed in nonlinear adaptation of fuzzy systems [15].
A similar nonlinear adaptation procedure was applied to the remaining 11 input variables of the institutional CLP model. Then, the MFs of the institutional context model were replaced with the adapted ones, resulting in the nonlinearly adapted institutional context model for use in industrial context. The nonlinearly adapted CLP model was used to predict CLP values of the industrial context, which had 23 data instances (refer to Table 1). The adapted model had an initial $\mathrm{RMSE}_{\mathrm{CA}-\mathrm{NL}}$ value of 2.742 .

4.3. Sensitivity Analysis of Adapted Models for Fuzzy Operators and Defuzzification Methods. The sensitivity of the linearly and nonlinearly adapted CLP models was then further evaluated by changing the fuzzy operators and defuzzification methods. The following options of fuzzy operators and defuzzification methods were tested: for input aggregation [MIN (minimum) and PROD (product)], for implication [MIN (minimum) and PROD (product)], for rule aggregation [MAX (maximum), SUM (sum of each rule's output set), and PROBOR (probabilistic OR)], and for defuzzification [CENTROID, BISECTOR, MOM (middle of maximum), LOM (largest of maximum), and SOM (smallest of maximum)]. The sensitivity options were varied one at a time, and a total of 30 unique combinations were tested. The options and results for linearly adapted institutional context model, which was linearly adapted to suit industrial context, are shown in Table 2.

For each linear and nonlinear adapted CLP model, the final adapted model's (i.e., after sensitivity analysis) accuracy measures of RMSE $\mathrm{CA}_{\mathrm{CAS}}$ and RMSE $\mathrm{CA}_{\mathrm{CLS}}$ of 1.832 and 2.738, respectively, were determined based on the best combination of the listed options of fuzzy operators and defuzzification methods. The results yielding the lowest $\mathrm{RMSE}_{\mathrm{CA}-\mathrm{LS}}$ and RMSE $_{\text {CA-NLS }}$ values, shown in bold in Table 2, provided the best adapted fuzzy operators and defuzzification methods for adapted CLP models. Finally, the agreement index $\mathrm{WI}_{i}$ between the adapted and the base model, described in Table 3, was computed using the modified Willmott index (see (5)). Accordingly, the appropriate context adaptation approach was the linearly adapted model, as it had an agreement index of 0.340 , while the nonlinearly adapted model had an agreement index of only 0.142 .

\section{Results and Discussion}

The linear and nonlinear context adaptation framework was tested and validated using the four context-specific CLP models, as shown in Figure 3, by comparing the prediction results of the context adapted models with the actual field data of the new context. The results of the context adaptation process, summarized for each context, are presented in the following sections.

5.1. Industrial Context CLP Models. For the industrial context, six adapted models were developed from warehouse, high-rise, and institutional contexts based on linear and nonlinear adaptation process. The base CLP model for industrial context had an RMSE value of 1.162. In Table 3 

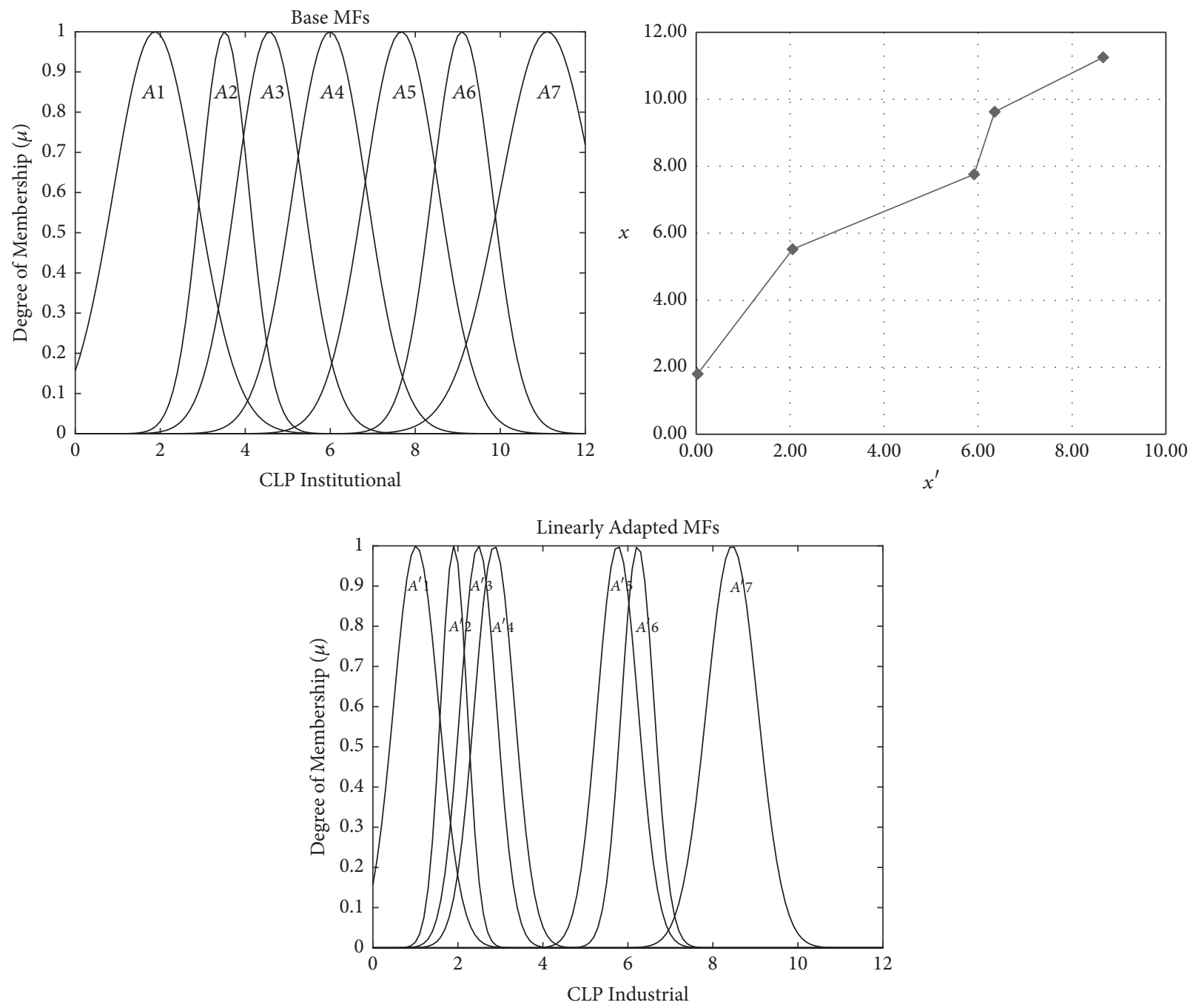

FIGURE 6: Nonlinear context adaptation of CLP feature from institutional to industrial context.

the results of the adaptation process are shown, where the initial RMSE values represent the accuracy of the adapted models in predicting the CLP values of the Industrial context, and the final RMSE values represent the accuracy of the adapted models after sensitivity analysis. The best performing fuzzy operators and defuzzification methods are also shown in Table 3.

Based on the agreement indices of the six adapted models, as shown in Table 3, the model linearly adapted from institutional context has the highest agreement with the base model of the industrial context with an agreement index value of 0.340 and RMSE value of 1.832 . The model nonlinearly adapted from institutional context has the least agreement with the base model with an agreement index value of 0.142 and RMSE value of 2.738. Additionally, the linearly adapted models from all three contexts, as compared to the nonlinearly adapted models, had better agreement indices with the base model; the models also had a higher accuracy based on RMSE values. However, it should be noted that, in terms of model accuracy, none of the adapted models performed better than the base model.

5.2. Warehouse Context CLP Models. For the warehouse context, six adapted models were developed from industrial, high-rise, and institutional contexts and based on linear and nonlinear adaptation process. The base CLP model for warehouse context had an RMSE value of 0.467 . Based on the agreement indices of the six adapted models, the model nonlinearly adapted from industrial context has the highest agreement to the base model of the warehouse context with an agreement index value of 0.459 and RMSE value of 0.939 . The model linearly adapted from institutional context has the least agreement with the base model with an agreement index value of 0.144 and RMSE value of 2.267. Additionally, the nonlinearly adapted models from industrial and highrise contexts, as compared to respective the linear adapted models, had better agreement with the base model; the models also had a higher accuracy based on RMSE values. 
TABLE 2: Context adapted models' sensitivity analysis: linearly and nonlinearly adapted institutional model.

\begin{tabular}{|c|c|c|c|c|c|c|}
\hline \multirow[b]{2}{*}{ Case } & \multicolumn{6}{|c|}{ Fuzzy operators and defuzzification methods } \\
\hline & Input aggregation & $\begin{array}{l}\text { Implication } \\
\text { method }\end{array}$ & Rule aggregation & $\begin{array}{l}\text { Defuzzification } \\
\text { method }\end{array}$ & $\begin{array}{c}\text { Linearly adapted } \\
\text { model accuracy } \\
\left(\mathrm{RMSE}_{\mathrm{CA}-\mathrm{LS}}\right)\end{array}$ & $\begin{array}{c}\text { Nonlinearly adapted } \\
\text { model accuracy } \\
\left(\mathrm{RMSE}_{\mathrm{CA}-\mathrm{NLS}}\right)\end{array}$ \\
\hline 1 & MIN & MIN & MAX & CENTROID & 1.840 & 2.742 \\
\hline 2 & MIN & MIN & MAX & BISECTOR & 1.840 & 2.742 \\
\hline 3 & MIN & MIN & MAX & MOM & 1.955 & 2.766 \\
\hline 4 & MIN & MIN & MAX & LOM & 2.030 & 2.762 \\
\hline 5 & MIN & MIN & MAX & $\mathrm{SOM}$ & 1.955 & 2.766 \\
\hline 6 & MIN & MIN & SUM & CENTROID & 1.840 & 2.742 \\
\hline 7 & MIN & MIN & SUM & BISECTOR & 1.840 & 2.742 \\
\hline 8 & MIN & MIN & SUM & MOM & 1.955 & 2.766 \\
\hline 9 & MIN & MIN & SUM & LOM & 2.030 & 2.762 \\
\hline 10 & MIN & MIN & SUM & SOM & 1.955 & 2.766 \\
\hline 11 & MIN & MIN & PROBOR & CENTROID & 1.840 & 2.742 \\
\hline 12 & MIN & MIN & PROBOR & BISECTOR & 1.840 & 2.742 \\
\hline 13 & MIN & MIN & PROBOR & MOM & 1.955 & 2.766 \\
\hline 14 & MIN & MIN & PROBOR & LOM & 2.030 & 2.762 \\
\hline 15 & MIN & MIN & PROBOR & SOM & 1.955 & 2.766 \\
\hline 16 & PROD & PROD & MAX & CENTROID & 1.832 & 2.738 \\
\hline 17 & PROD & PROD & MAX & BISECTOR & 1.840 & 2.740 \\
\hline 18 & PROD & PROD & MAX & MOM & 1.955 & 2.765 \\
\hline 19 & PROD & PROD & MAX & LOM & 2.030 & 2.762 \\
\hline 20 & PROD & PROD & MAX & SOM & 1.955 & 2.765 \\
\hline 21 & PROD & PROD & SUM & CENTROID & 1.832 & 2.738 \\
\hline 22 & PROD & PROD & SUM & BISECTOR & 1.840 & 2.740 \\
\hline 23 & PROD & PROD & SUM & MOM & 1.840 & 2.740 \\
\hline 24 & PROD & PROD & SUM & LOM & 2.030 & 2.762 \\
\hline 25 & PROD & PROD & SUM & SOM & 1.955 & 2.765 \\
\hline 26 & PROD & PROD & PROBOR & CENTROID & 1.832 & 2.738 \\
\hline 27 & PROD & PROD & PROBOR & BISECTOR & 1.840 & 2.742 \\
\hline 28 & PROD & PROD & PROBOR & MOM & 1.955 & 2.766 \\
\hline 29 & PROD & PROD & PROBOR & LOM & 2.030 & 2.762 \\
\hline 30 & PROD & PROD & PROBOR & SOM & 1.955 & 2.766 \\
\hline
\end{tabular}

However, in terms of model accuracy, none of the adapted models performed better than the base model.

5.3. High-Rise Context CLP Models. For the high-rise context, six adapted models were developed from industrial, warehouse, and institutional contexts and based on linear and nonlinear adaptation process. The base or base CLP model for high-rise context had an RMSE value of 0.992. Based on the agreement indices of the six adapted models, the model nonlinearly adapted from warehouse context has the highest agreement to the base model of the high-rise context with an agreement index value of 0.427 and RMSE value of 3.851. The model linearly adapted from institutional context has the least agreement with the base model with an agreement index value of 0.181 and RMSE value of 4.627. Additionally, the nonlinearly adapted models from all three contexts, as compared to the linearly adapted models, had better or equal agreement with the base model. However, both linearly and nonlinearly adapted models had similar accuracy based on RMSE values. Similar to the contexts discussed above, none of the adapted models performed better than the base model.

5.4. Institutional Context CLP Models. For the institutional context, six adapted models were developed from industrial, warehouse, and high-rise contexts and based on linear and nonlinear adaptation process. The base CLP model for institutional context had an RMSE value of 0.671 . Based on the agreement indices of the six adapted models, the model linearly adapted from industrial context has the highest agreement to the base model of the institutional context with an agreement index value of 0.398 and RMSE value of 2.552. The model nonlinearly adapted from industrial context has 
TABLE 3: Context adaptation results for industrial context (C1).

\begin{tabular}{|c|c|c|c|c|c|c|}
\hline \multirow[t]{2}{*}{ Adapted models } & \multicolumn{2}{|c|}{ Adapted from warehouse: C2 } & \multicolumn{2}{|c|}{ Adapted from high-rise: C3 } & \multicolumn{2}{|c|}{$\begin{array}{l}\text { Adapted from institutional: } \\
\text { C4 }\end{array}$} \\
\hline & Linear & Nonlinear & Linear & Nonlinear & Linear & Nonlinear \\
\hline RMSE (initial) & 1.892 & 2.079 & 1.880 & 1.880 & 1.832 & 2.742 \\
\hline RMSE (final) & 1.831 & 2.028 & 1.873 & 1.873 & 1.832 & 2.738 \\
\hline $\begin{array}{l}\text { Sensitivity } \\
\text { improvement (\%) }\end{array}$ & 3.26 & 2.47 & 0.32 & 0.32 & 0.00 & 0.14 \\
\hline Agreement index & 0.203 & 0.188 & 0.282 & 0.282 & 0.340 & 0.142 \\
\hline $\begin{array}{l}\text { Input aggregation } \\
\text { operator }\end{array}$ & PROD & PROD & PROD & PROD & PROD & PROD \\
\hline $\begin{array}{l}\text { Implication } \\
\text { method }\end{array}$ & PROD & PROD & PROD & PROD & PROD & PROD \\
\hline $\begin{array}{l}\text { Rule aggregation } \\
\text { operator }\end{array}$ & SUM & MAX & PROBOR & PROBOR & PROBOR & PROBOR \\
\hline $\begin{array}{l}\text { Defuzzification } \\
\text { method }\end{array}$ & BISECTOR & MOM & MOM & MOM & CENTROID & CENTROID \\
\hline
\end{tabular}

the least agreement with the base model with an agreement index value of 0.199 and RMSE value of 2.552. However, a clear difference between linear and nonlinear adaptation options based on agreement or accuracy measures were not found in the institutional context case. Also, in terms of model accuracy, none of the adapted models performed better than the base model.

In summary, the review of the linear and nonlinear adaptation approaches indicated that linear adapted models were in better agreement with those of the base models for industrial and institutional contexts, and nonlinear adapted models were in better agreement with those of the base models for warehouse and high-rise contexts. However, the use of nonlinear adaptation approach has resulted in reduced interpretability of the adapted models, due to the change in the shape and distribution and higher overlap of adapted MFs. Notably, the sensitivity analysis on fuzzy operators and defuzzification methods did not show significant improvement in adapted model's accuracy. This finding supports the option used in past studies, such as Botta [3], which only used minimum operator for input aggregation and implication and centroid for defuzzification.

In terms of prediction accuracy, in all four contexts, none of the adapted models performed better than the base models. This is expected as the base models have been developed and further optimized to improve models' accuracy [7]. The comparison of the adapted models' accuracy with those of the base context-specific models, before optimization process was carried out to fine tune the base model's parameters as described in Tsehayae and Fayek [7], showed promising results for the industrial and warehouse contexts. The base industrial context model before optimization had an RMSE value of 1.582, and the most accurate linearly adapted model from warehouse context has an RMSE value of 1.831. Similarly, the base warehouse context model before optimization had an RMSE value of 0.586 and the most accurate linearly adapted model from high-rise context has an RMSE value of 0.719.
Considering the effort and cost required for collecting data on all CLP influencing variables and to develop and optimize new models, the use of context adaptation framework presented in this paper, which enables the reuse of existing CLP models through the transfer of knowledge from one context to another will provide a simpler and efficient alternative for developing CLP models.

\section{Conclusions and Future Research}

Construction labor productivity is one of the most studied areas in the construction research field, and several-FISbased predictive models have been developed; however, a framework for adapting such FIS-based models from one context to another is missing. This paper developed a context adaptation framework for transferring the knowledge represented in FIS-based CLP models from one context to another. The framework employed linear and genetic optimizationbased nonlinear MF transformation functions accompanied by sensitivity analysis for fuzzy operators and defuzzification methods of the adapted FIS models. Using four contextspecific CLP models for concreting activity under industrial, warehouse, high-rise, and institutional building contexts, the developed context adaptation framework was implemented. Comparisons of the adapted models, using agreement indices and RMSE accuracy measures, with the base model of the four contexts uncovered many findings. The results of the investigation indicated that linearly adapted CLP models were similar to the base CLP models in industrial and institutional contexts, while the nonlinearly adapted CLP models were similar to the base CLP models in warehouse and high-rise contexts. However, in terms of model accuracy, none of the adapted models performed better than the base model of a given context. Additionally, the sensitivity analysis on fuzzy operators and defuzzification methods did not show significant improvement in adapted model accuracy. Furthermore, the best adapted model for each 
context was validated and contextual similarities in terms of CLP prediction agreement indices between base and adapted models were examined.

The contributions of this paper can be grouped into three areas. First, the paper showed the value of context adaptation approaches using a practical context-sensitive application problem (CLP modeling). While past studies manly focused on demonstrating the value of context adaptation approaches using benchmark datasets and theoretical context independent MFs defined over a normalized universe of discourse, this study tested the context adaptation approaches using real world CLP datasets collected from six construction projects and context-dependent MFs defined over the actual universes of discourses of FIS model features. This study also investigated the effect of fuzzy operators and defuzzification methods in the context adaptation of FIS and showed their limited effect on the accuracy of the adapted models. Second, the paper has advanced the state of the art in fuzzy modeling in CLP studies by introducing and testing an approach for adapting MF using linear and genetic optimizationdriven nonlinear transformation functions, which provided a transparent approach for adapting MFs from base to adapted contexts. Finally, the paper developed a context adaptation framework, which enables researchers and industry practitioners to take full advantage of existing FIS-based CLP models in the study of new contexts for which data availability is limited. The framework also improves the application of existing CLP models by construction companies as existing models can be adapted to suit the companies' specific need or context. Also, the comparison of the adapted and original models demonstrated that, for the sake of CLP model development, similarities among unique construction contexts do exist. Such similarities will assist project managers to easily transfer past experiences and knowledge bases and lead to the better management of future projects.

Currently, additional data are being collected from ongoing construction projects to further test this study and its findings and to examine other nonlinear transformation functions so as to improve the prediction accuracy of adapted CLP models. Future research is focusing on the development of a framework for abstraction of various context-specific CLP models to develop a universal or granular CLP model capable of predicting CLP in any context. This approach will explore the practicality and performance of a variety of granular approaches, including case-based reasoning, fuzzy clustering, and fuzzy regression, to identify and aggregate the most important relationships represented in the contextspecific models and produce a universal model that is better able to predict CLP.

\section{Data Availability}

All data generated or analyzed during the study are included in the submitted article.

\section{Conflicts of Interest}

The authors declare that there are no conflicts of interest regarding the publication of this paper.

\section{Acknowledgments}

The authors would like to express their sincere appreciation to all of the companies that participated in this study for their cooperation, time, and the valuable information they provided. The authors particularly recognize the graduate research assistants who diligently helped with data collection. This research was conducted under the NSERC Industrial Research Chair in Strategic Construction Modeling and Delivery (NSERC IRCPJ 428226-15), held by Dr. Aminah Robinson Fayek. The financial support of the industrial partners to this chair and of the Natural Sciences and Engineering Research Council of Canada is gratefully acknowledged.

\section{References}

[1] W. Yi and A. Chan, "Critical review of labor productivity research in construction journals," Journal of Management in Engineering, vol. 30, no. 2, pp. 214-225, 2014.

[2] K. M. El-Gohary, R. F. Aziz, and H. A. Abdel-Khalek, "Engineering approach using ANN to improve and predict construction labor productivity under different influences," Journal of Construction Engineering and Management, vol. 143, no. 8, Article ID 04017045, 2017.

[3] A. Botta, Automatic context adaptation of fuzzy systems [Ph.D. thesis], IMT Institute for Advanced Studies, Lucca, Italy, 2008.

[4] H. Mao, Estimating labour productivity using fuzzy set theory [M.Sc. thesis], University of Alberta, Edmonton, Alberta, Canada, 1999.

[5] N.-F. Pan, "Assessment of productivity and duration of highway construction activities subject to impact of rain," Expert Systems with Applications, vol. 28, no. 2, pp. 313-326, 2005.

[6] A. R. Fayek and A. Oduba, "Predicting industrial construction labor productivity using fuzzy expert systems," Journal of Construction Engineering and Management, vol. 131, no. 8, pp. 938-941, 2005.

[7] A. A. Tsehayae and A. R. Fayek, "Developing and Optimizing Context-Specific Fuzzy Inference System-Based Construction Labor Productivity Models," Journal of Construction Engineering and Management, vol. 142, no. 7, Article ID 4016017, 2016.

[8] A. Botta, B. Lazzerini, F. Marcelloni, and D. C. Stefanescu, "Context adaptation of fuzzy systems through a multi-objective evolutionary approach based on a novel interpretability index," Soft Computing, vol. 13, no. 5, pp. 437-449, 2009.

[9] O. Cordón, F. Herrera, L. Magdalena, and P. Villar, "A genetic learning process for the scaling factors, granularity and contexts of the fuzzy rule-based system data base," Information Sciences, vol. 136, no. 1-4, pp. 85-107, 2001.

[10] W. Pedrycz, R. R. Gudwin, and F. A. C. Gomide, "Nonlinear context adaptation in the calibration of fuzzy sets," Fuzzy Sets and Systems, vol. 88, no. 1, pp. 91-97, 1997.

[11] M. Engwall, "No project is an island: Linking projects to history and context," Research Policy, vol. 32, no. 5, pp. 789-808, 2003.

[12] R. R. Gudwin, F. A. Gomide, and W. Pedrycz, "Context adaptation in fuzzy processing and genetic algorithms," International Journal of Intelligent Systems, vol. 13, no. 10-11, pp. 929-948, 1998.

[13] R. R. Gudwin and F. Gomide, "Context adaptation in fuzzy processing," in Proceedings of Brazil-Japan Joint Symposium on Fuzzy Systems, Campinas, Brazil, 1994. 
[14] L. Magdalena, "Adapting the gain of an FLC with genetic algorithms," International Journal of Approximate Reasoning, vol. 17, no. 4, pp. 327-349, 1997.

[15] D. T. Ho, Context dependent fuzzy modelling and its application [Ph.D. thesis], University of Nottingham, Nottingham, UK, 2013.

[16] W. Pedrycz and F. Gomide, Fuzzy Systems Engineering: toward Human-Centric Computing, John Wiley and Sons, Hoboken, New Jersey, US, 2007.

[17] P. Pulkkinen and H. Koivisto, "A dynamically constrained multiobjective genetic fuzzy system for regression problems," IEEE Transactions on Fuzzy Systems, vol. 18, no. 1, pp. 161-177, 2010.

[18] L. Magdalena, "On the role of context in hierarchical fuzzy controllers," International Journal of Intelligent Systems, vol. 17, no. 5, pp. 471-493, 2002.

[19] G. Klir and B. Yuan, Fuzzy Sets and Fuzzy Logic, Prentice Hall, Upper Saddle River, New Jersey, US, 1995.

[20] A. Awad and A. R. Fayek, "Adaptive learning of contractor default prediction model for surety bonding," Journal of Construction Engineering and Management, vol. 139, no. 6, pp. 694704, 2013.

[21] A. Idrus, M. F. Fadhil Nuruddin, and M. A. Rohman, "Development of project cost contingency estimation model using risk analysis and fuzzy expert system," Expert Systems with Applications, vol. 38, no. 3, pp. 1501-1508, 2011.

[22] S. S. S. Ahmad, S. Ahmad, and W. Pedrycz, "Data and feature reduction in fuzzy modeling through particle swarm optimization," Applied Computational Intelligence and Soft Computing, vol. 10, pp. 1-21, 2012.

[23] C. Willmott, S. Robeson, and K. Matsuura, "A refined index of model performance," International Journal of Climatology, vol. 32, no. 13, pp. 2088-2094, 2012.

[24] A. A. Tsehayae, Developing and optimizing context-specific and universal construction labour productivity models [Ph.D. thesis], University of Alberta, Edmonton, Canada, 2015. 


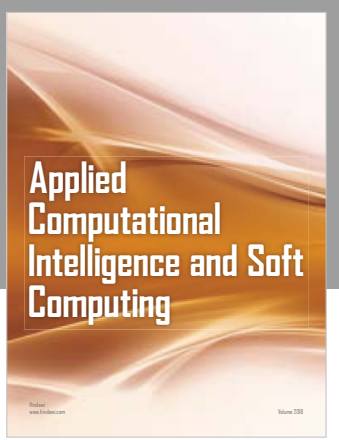

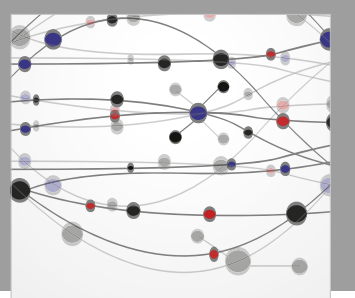

The Scientific World Journal
Submit your manuscripts at

Computing
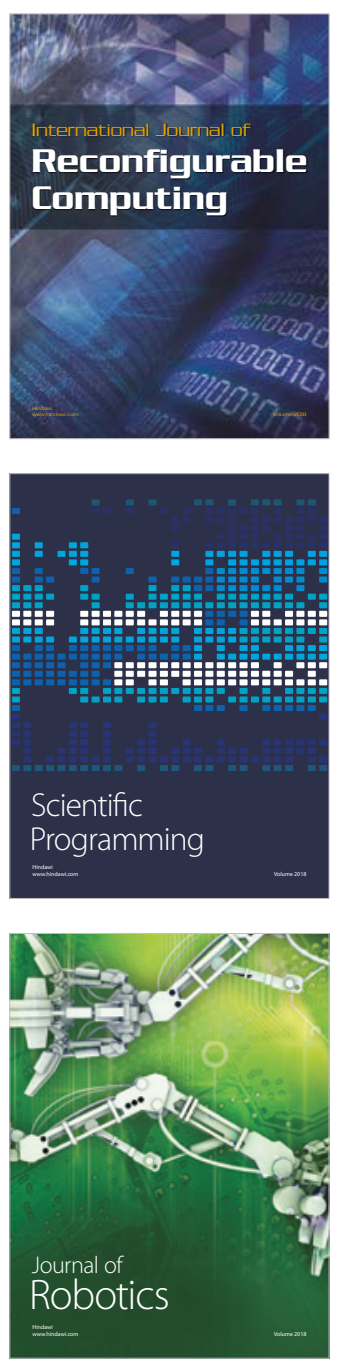

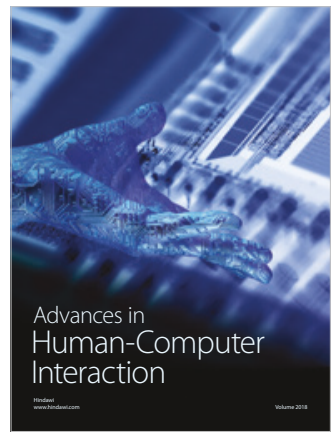

Human-Compute

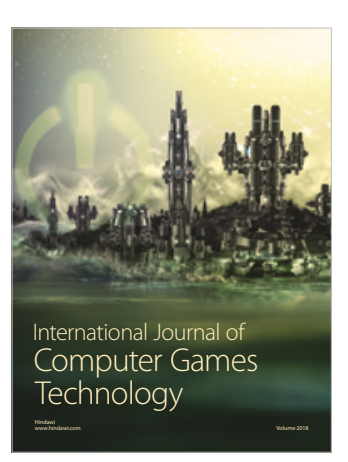

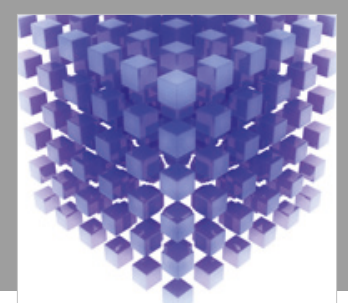

Mathematical Problems in Engineering

\section{Engincering}
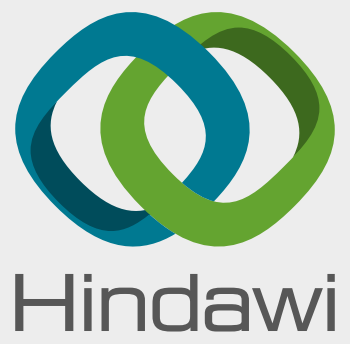

www.hindawi.com
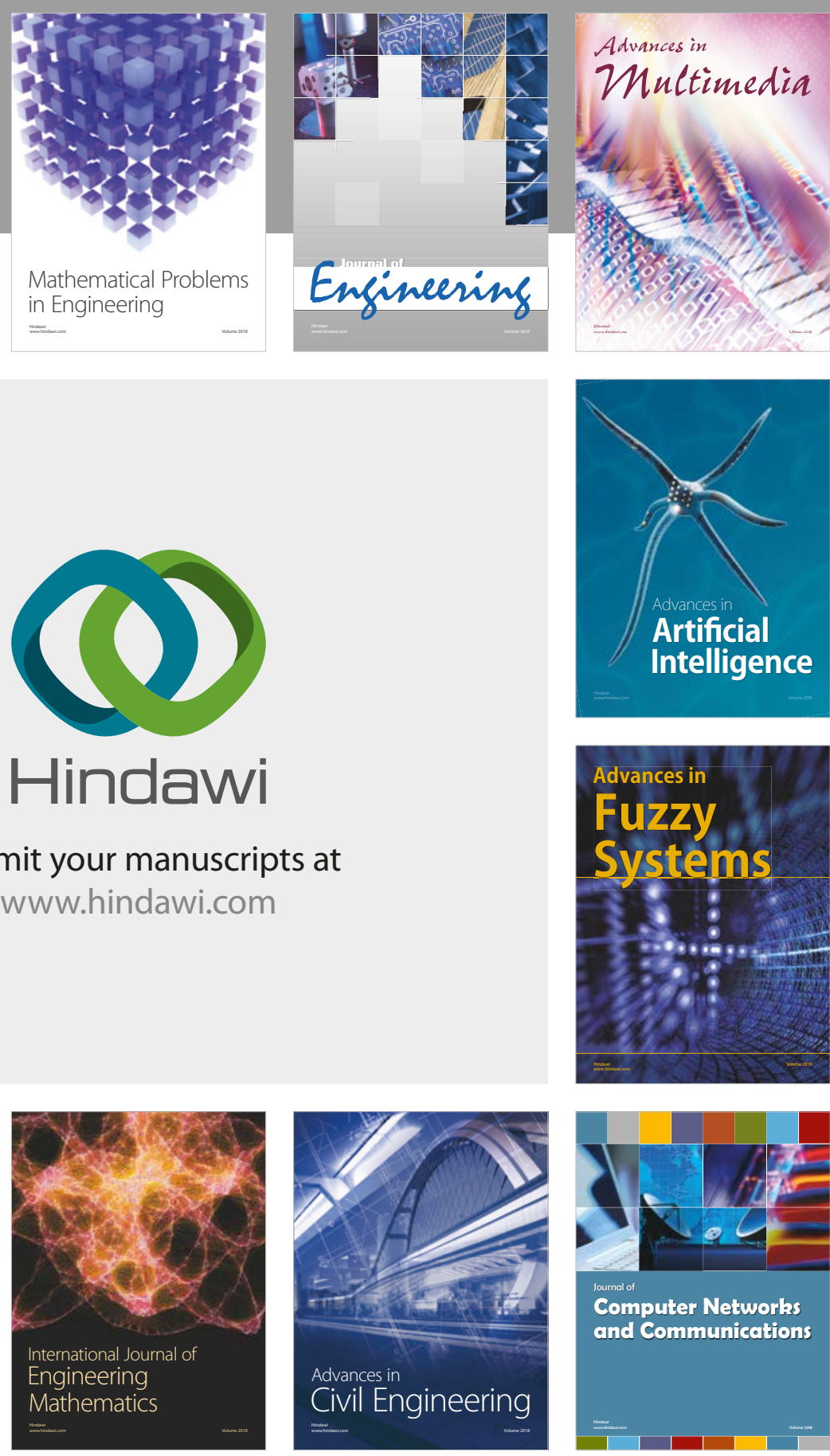

Computer Networks and Communications

Multimedia
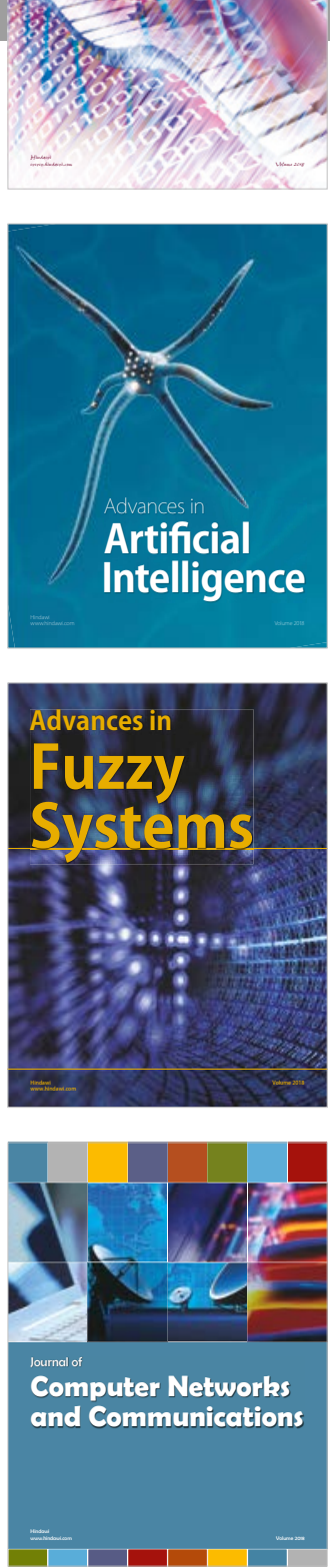

Advances in

Modelling \&

Simulation

in Engineering

interaction

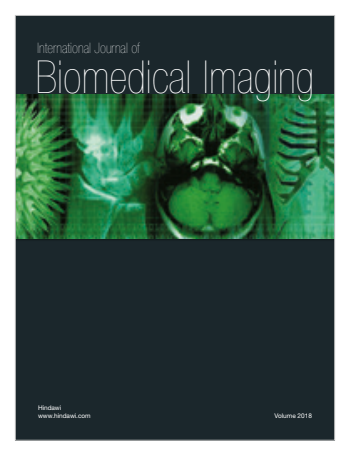

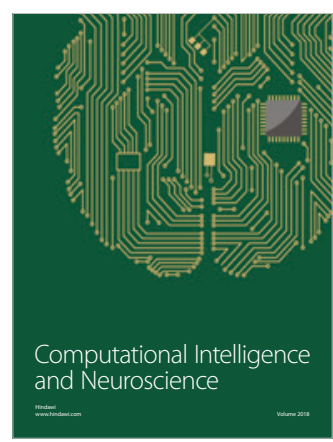

